\title{
Extensive Dermatosis Neglecta with Severe Atopic Dermatitis: A Case that Presented as a Suicide Attempt
}

\author{
Ryo Yoshinaga*, Yusuke Goto and Eiichi Tahara \\ Department of Japanese Oriental Medicine, Iizuka Hospital, 3-83 Yoshio-machi, Iizuka City, Japan
}

\begin{abstract}
In this report, we present a case of extensive dermatosis neglecta with severe atopic dermatitis which presented as a suicide attempt. A 19-year-old woman was admitted for a suicide attempt by drug overdose. Her body was almost entirely covered by dark brown plaque. During her recovery, she reported dermatological pain and hyperesthesia. She had suffered from atopic dermatitis (AD) since her childhood. She had stopped topical steroid therapy (TST) for fears of the side effects since becoming a teenager. She became socially withdrawn due to worsening AD and felt depressive. She had not bathed or showered for seven years and the plaque had developed over two years. She was diagnosed with depression and dermatosis neglecta with severe AD. She had emotional instability and strongly refused to receive TST. We started counselling from a psychiatrist and a Japanese traditional herbal (Kampo) medicine, Shofusan for her skin, which became a stepping-stone to undertaking and restarting TST. Her mental and dermatological conditions gradually improved. She continued these therapies as an outpatient. One year and ten months later, her skin had almost fully recovered, and she remained healthy. This case shows that severe AD and a misunderstanding of TST could contribute to extensive dermatosis neglecta. Comprehensive and integrated care was useful for the patient, who had depression with suicidal ideation and steroid phobia.
\end{abstract}

Abbreviations and symbols: AD: Atopic dermatitis; TST: Topical steroid therapy.

\section{Background}

Dermatosis neglecta, also known as unwashed dermatosis, is a condition secondary to lack of cleanliness, characterized by the formation of hyperkeratotic plaques located in a specific region of the body [1]. The diagnosis is clinical, therefore proper history taking is important and histopathology is not necessary [2,3]. We experienced a case of extensive dermatosis neglecta with severe atopic dermatitis (AD) which presented as a suicide attempt.

\section{Case}

A 19-year-old woman, whose body was almost entirely covered by dark brown plaque, was admitted for a suicide attempt by drug overdose. She had suffered from AD since her childhood. She had stopped topical steroid therapy (TST) for fears of the side effects since becoming a teenager and received some alternative remedies. She became socially withdrawn due to worsening $\mathrm{AD}$ and felt depressive, expressing suicidal tendencies. She affirmed that she had not bathed or showered for seven years because of her skin symptoms and the plaque had developed over two years. During her recovery from drug overdose, she reported dermatological pain and hyperesthesia. Due to these symptoms, her emotions were unstable, and she couldn't assume a decubitus or sitting position, spending almost all day in a standing position. On dermatological examination, there was erythema and exudate on her face, neck, and arms. Most of her body under the neck was covered by moist and thick hyperpigmented plaque except for her face, hands, and soles (Figures 1a and 1b). She disliked being touched on her body. We tried to wash and remove a part of the plaque under sedation. The plaque proved to be a hard and thick crust. A blood test showed RIST IgE of $77612 \mathrm{U} / \mathrm{ml}$. Her psychological state when evaluated with the Zung Self-rating Depression Scale (SDS) [4] was 60. She was diagnosed with depression and dermatosis neglecta associated with severe AD. She had emotional instability and strongly refused to receive TST. She started to receive counselling from a psychiatrist. In addition, we prescribed a Japanese traditional herbal (Kampo) medicine, shofusan referring to the condition of her skin. One week later, erythema and exudate of her skin improved slightly, and the alternative herbal therapy became a stepping-stone to undertaking and restarting TST. Two weeks later, she could sit down on the bed. And she expressed positive feelings and started taking a daily bath. Her mental conditions improved and the plaque on her body also progressively dissolved and peeled away. She was discharged after two months and continued these therapies as an outpatient. Six months later, her RIST IgE decreased to 33712, her skin condition improved (Figures $2 \mathrm{a}$ and $2 \mathrm{~b}$ ) and she could enjoy shopping and reading books in the library. Nine months later, her RIST IgE further decreased to 28624. One year and ten months later, her skin had almost fully recovered (Figure 3 ) and her SDS score improved to 49. She remained healthy, becoming a university student.

\section{Discussion}

The course of this patient provides two important clinical suggestions. First, extensive dermatosis neglecta can present as a complication of severe AD and steroid phobia. Second, comprehensive, and integrated care was useful for the patient, who had depression with suicidal ideation and steroid phobia.

${ }^{*}$ Correspondence to: Ryo Yoshinaga, Department of Japanese Oriental Medicine, Iizuka Hospital, 3-83 Yoshio-machi, Iizuka, Fukuoka 820-8505, Japan, Tel: +81948-22-3800; Fax: +81-948-29-8075; E-mail: ryoshinagah2@aih-net.com

Key words: dermatosis neglecta, atopic dermatitis, steroid phobia, depression, traditional herbal medicine

Received: August 07, 2020; Accepted: August 20, 2020; Published: August 24, 2020 


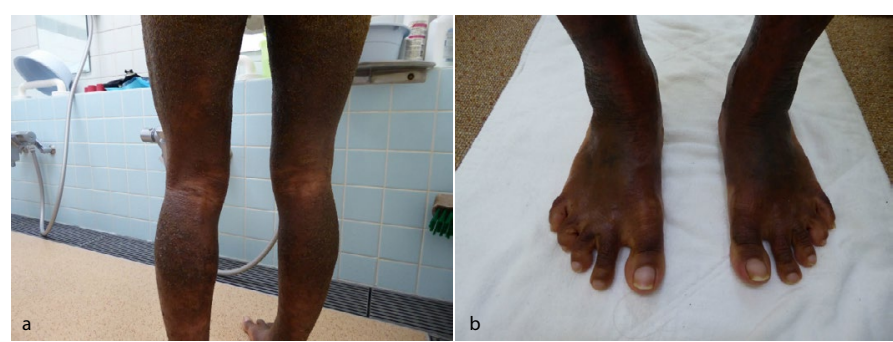

Figure 1. (a) Posterior surface of lower extremities just after admission (b) Dorsum of feet just after admission

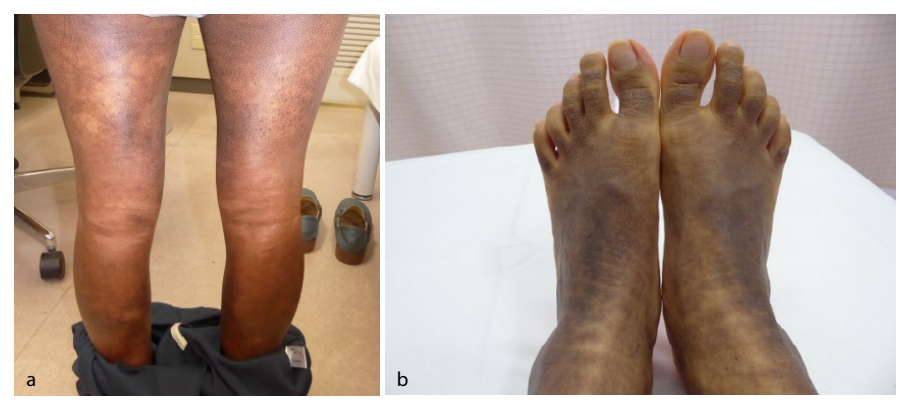

Figure 2. (a) Posterior surface of lower extremities two months after initiating the comprehensive therapies (b) Dorsum of feet two months after initiating the comprehensive therapies

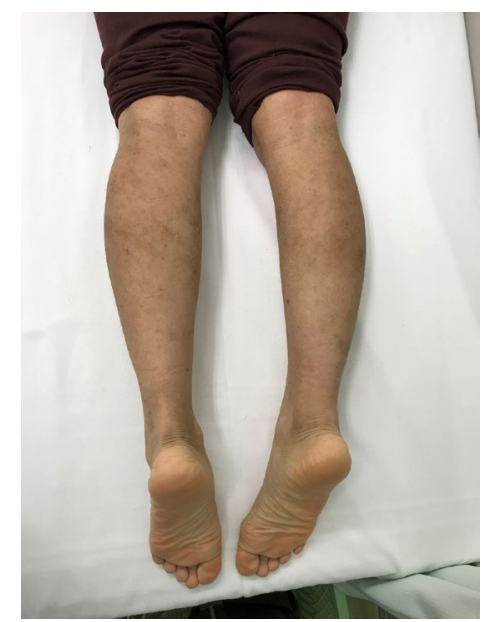

Figure 3. Posterior surface of lower extremities one year and ten months after

Several cases of localized dermatosis neglecta caused by physical disability, neurological deficit, and psychiatric condition have been reported $[2,3,5,6]$. To the best of our knowledge, this is the first report to show that severe AD and a misunderstanding of TST could contribute to extensive dermatosis neglecta. Adequate hygiene measures of cleaning with soap and water and by alcohol-soaked gauze are sufficient treatments for the majority of patients with dermatosis neglecta $[2,3]$. Due to dermatological pain and hyperesthesia, which were considered to be complications of $\mathrm{AD}$ and her mental condition, it was difficult to wash or scrub her skin which took a long time to recover.

The second clinical suggestion in this case is that comprehensive and integrated care including psychiatric counseling and traditional herbal medicine was useful for the patient, who had depression with suicidal ideation and steroid phobia. AD is a common chronic skin disease which often develops in childhood. Patients with AD are at risk of psychiatric conditions such as anxiety, depression, and suicidal ideation [7-9]. In addition, poor adherence to treatment for $\mathrm{AD}$ is a major limiting factor in its outcome. Some important reasons for treatment failures are fear of side effects, lack of understanding of disease or treatment, and lack of trust in the physician [10]. The prevalence of steroid phobia was reported to be from $21.0 \%$ to $83.7 \%$ in patients with $\mathrm{AD}$ [11]. In Japan, traditional herbal (Kampo) medicine is covered by national health insurance and integrated into modern medicine. Some Kampo formulas including Shofusan can been seen in Japanese guidelines for $\mathrm{AD}$ and utilized as an adjunctive therapy [12]. Shofusan, which was named as xiao-feng-san in traditional Chinese medicine, is a representative formulation for the chronic dermatitis which causes itching, erythema, and swelling skin lesions with discharge [13]. A past randomized, double-blind controlled trial shows the effectiveness of xiao-feng-san for refractory AD [14]. Furthermore, the proportion of children with $\mathrm{AD}$ receiving alternative therapies whose parents have steroid phobia is higher than in those whose parents don't in a past study [15]. We suggest traditional herbal medicine, which can be utilized in combination with the standard therapies, could be a factor in reducing steroid phobia and ultimately benefit the physician-patient relationship.

In conclusion, we experienced a case of extensive dermatosis neglecta with severe $\mathrm{AD}$ which presented as a suicide attempt. Comprehensive and integrated care was useful for the patient, who had depression and steroid phobia. Traditional herbal medicine could become a bridge between standard therapies and those with strong fears of western medicine.

\section{Declarations}

\section{Authorship and contributions}

This manuscript has not been published and is not under consideration for publication elsewhere. All authors have contributed significantly, and author contributions are as follows; All authors performed clinical assessments and date curation. Ryo Yoshinaga (R.Y.) and Yusuke Goto (Y.G.) wrote the manuscript. R.Y. and Eiichi Tahara (E.T.) were involved in revising the manuscript. R.Y. and Y.G. were in charge of visualization. E.T. were involved in supervision. All the authors have read the final version of the manuscript and have approved for this submission.

\section{Informed consent}

We have obtained written informed consent from the patient for publication of this case report.

\section{Acknowledgements}

We thank the patient for granting permission to publish this information.

\section{Funding information}

None.

\section{Competing interests}

Ryo Yoshinaga and Eiichi Tahara have received lecture fees from Tsumura \& Co.

\section{Supplemental material}

Shofusan, which we used in this case consists of Sophorae Radix 4 g, Schizonepetae Spica 3 g, Arctii Fructus 3 g, Sesami Semen 1.5 g, Rehmanniae Radix 3 g, Gypsum Fibrosum 20 g, Cicadae Periostracum $1 \mathrm{~g}$, Atractylodis Lanceae Rhizoma $8 \mathrm{~g}$, Anemarrhenae Rhizoma $1.5 \mathrm{~g}$, Angelicae Radix 1 g, Ledebouriellae Radix 2 g, and Akebiae Caulis 2 g. The decoction was prepared by boiling the crude drugs in $600 \mathrm{ml}$ of 
water for 40 minutes until the volume of the solution was concentrated to $300 \mathrm{ml}$. The decoction was administered 3 times a day.

\section{References}

1. Poskitt L, Wayte J, Wojnarowska F, Wilkinson JD (1995) 'Dermatitis neglecta': unwashed dermatosis. Br J Dermatol 132: 827-829.

2. Lopes S, Vide J, Antunes I, Azevedo F (2018) Dermatitis neglecta: a challenging diagnosis in psychodermatology. Acta Dermatovenerol Alp Pannonica Adriat 27: 109110. [Crossref]

3. Perez-Rodriguez IM, Munoz-Garza FZ, Ocampo-Candiani J (2014) An unusually severe case of dermatosis neglecta: a diagnostic challenge. Case Rep Dermatol 6: 194199. [Crossref]

4. Zung WW (1965) A Self-rating depression scale. Arch Gen Psychiatry 12: 63-70. [Crossref]

5. Saha A, Seth J, Sharma A, Biswas D (2015) Dermatitis neglecta -- a dirty dermatosis: report of three cases. Indian J Dermatol 60: 185-187. [Crossref]

6. Singh P, Kar SK, Kumari R, Gupta SK (2015) Dermatosis neglecta in schizophrenia: a rare case report. Indian J Psychol Med 37: 93-95. [Crossref]

7. Paller A, Jaworski JC, Simpson EL, Boguniewicz M, Russell JJ, et al. (2018) Major comorbidities of atopic dermatitis: Beyond allergic disorders. Am J Clin Dermatol 19: 821-838. [Crossref]
8. Sandhu JK, Wu KK, Bui TL, Armstrong AW (2019) Association between atopic dermatitis and suicidality: A systematic review and meta-analysis. JAMA Dermatol 155: 178-187. [Crossref]

9. Thyssen JP, Hamann CR, Linneberg A, Dantoft TM, Skov L, et al. (2018) Atopic dermatitis is associated with anxiety, depression, and suicidal ideation, but not with psychiatric hospitalization or suicide. Allergy 73: 214-220. [Crossref]

10. Patel NU, D'Ambra V, Feldman SR (2017) Increasing Adherence with topical agents for atopic dermatitis. Am J Clin Dermatol 18: 323-332. [Crossref]

11. Li AW, Yin ES, Antaya RJ (2017) Topical corticosteroid phobia in atopic dermatitis: A systematic review. JAMA Dermatology 153: 1036-1042. [Crossref]

12. Katayama I, Aihara M, Ohya Y, Saeki H, Shimojo N, et al. (2017) Japanese guidelines for atopic dermatitis 2017. Allergol Int 66: 230-247. [Crossref]

13. Chen YC, Lin YH, Hu S, Chen HY (2016) Characteristics of traditional Chinese medicine users and prescription analysis for pediatric atopic dermatitis: a populationbased study. BMC Complement Altern Med 16: 173. [Crossref]

14. Cheng HM, Chiang LC, Jan YM, Chen GW, Li TC (2011) The efficacy and safety of a Chinese herbal product (Xiao-Feng-San) for the treatment of refractory atopic dermatitis: a randomized, double-blind, placebo-controlled trial. Int Arch Allergy Immunol 155: 141-148. [Crossref]

15. Kojima R, Fujiwara T, Matsuda A, Narita M, Matsubara O, et al. (2013) Factors associated with steroid phobia in caregivers of children with atopic dermatitis. Pediatr Dermatol 30: 29-35. [Crossref]

Copyright: (C2020 Yoshinaga R. This is an open-access article distributed under the terms of the Creative Commons Attribution License, which permits unrestricted use, distribution, and reproduction in any medium, provided the original author and source are credited. 